\title{
Purification Capability of White Radish for Gaseous Ethyl Alcohol and Bioelectric Potential under LED Light
}

\author{
Takashi Oyabu*, Masanori Kawauchi ${ }^{1}$, Hiromi Ban ${ }^{2}$ and Hidehito Nanto ${ }^{3}$ \\ Kokusai Business Gakuin College, Minamicyo 6-12, Kanazawa, 920-0919 Japan \\ ${ }^{1}$ Kanazawa Kiko, Muryoji, Ha38-2, Kanazawa, 920-0332 Japan \\ ${ }^{2}$ Nagaoka University of Technology, Nagaoka, 940-2188 Japan \\ ${ }^{3}$ Kanazawa Institute of Technology, Yatsukaho, Hakusan, 924-0838 Japan
}

(Received January 20, 2014; accepted April 7, 2014)

Key words: bioelectric potential, LED, white radish, SPA, absorption capability

Bioelectric potential is caused by ion stream in vegetation tissue. This potential could be applied as a speaking plant approach (SPA) technique. In this study, the potential of white radish is measured in an ethyl alcohol atmosphere to establish the SPA technique. The absolute summation $\left(v_{\mathrm{m} 1}\right)$ of the potential for $1 \mathrm{~min}$ is adopted to analyze the potential characteristic, and the frequency of an LED panel and the ethyl alcohol concentration are adopted as parameters. Moreover, the absorption capability $\left(P_{\mathrm{a}}\right)$ of white radish for the alcohol is derived and its relationship with $v_{\mathrm{m} 1}$ is examined. As a result, it is clarified that there is a correlation between $v_{\mathrm{m} 1}$ and $P_{\mathrm{a}}$, namely, $P_{\mathrm{a}}$ increases with $v_{\mathrm{m} 1}$. The distribution of $P_{\mathrm{a}}$ in relation to the light frequency and concentration indicates a normal distribution, and it is considered that the potential is related to the growth rate of vegetation. Other summations $v_{\mathrm{m} 30}$ and $v_{\mathrm{h} 1}$ for $30 \mathrm{~min}$ and $1 \mathrm{~h}$ are also adopted to derive their relationship with the absorption capability $\left(P_{\mathrm{a}}\right)$ for the substance.

\section{Introduction}

Plants have various functions. A plant can sense the surrounding luminosity, odor, and even the touch of a person. Recently, it has been found that a plant has both sensory and memory functions. ${ }^{(1)}$ Plant capabilities can be recognized by studying various plant functions. A plant has the capability to purify soil, water, and airborne chemicals. ${ }^{(2-4)}$ It can grow by absorbing air-polluting substances, such as carbon dioxide, nitrogen oxide, and hydrocarbon, which are exhausted by cars. Vegetation also has the information transmission capability, for instance, bioelectric potential. ${ }^{(5-7)}$ There is a technology for gathering a lot of information from plants, which is called the speaking plant approach (SPA) in Japan, and the technique has been adopted in plant factories.

*Corresponding author: e-mail: oyabu24@gmail.com 
Plants have been produced as food since ancient times. Food self-sufficiency has become unbalanced owing to the rapid population increase on Earth. Therefore, plant factories, which can produce and provide vegetation as food, have received a lot of attention. Many vegetations produced in these factories are leafy vegetables such as lettuce. They have a high growth rate and market competitiveness because of their low unit price. One of the challenges in the plant factories is how to reduce the production cost. The production of a value-added vegetable in the factories contributes to the increase in food production. They have considered that the plant growth rate and fertilization period could be determined through the signal emitted by the plant, and a more efficient production in a vegetable factory will be achieved. Labor saving and cost reduction are accomplished in this factory. These are great advantages. ${ }^{(8)}$

In this study, the bioelectric potential of white radish is examined under eight irradiation light frequencies including darkness, and its absorption capability in an atmosphere of gaseous ethyl alcohol is also investigated. The potential is one of the important signals emitted by the plant. It is considered that plant growth and absorption capability for carbon dioxide differ depending on the irradiation light frequency. ${ }^{(9-11)}$ White radish is adopted as the subject plant. There was a record showing that it was an important food for laborers working in pre-Hispanic pyramids. It is known to nearly everyone and is also used as a medicinal plant. Its big root is eaten and it is a type of typical root crop. Ethyl alcohol in the atmosphere is used as a nutrient component. This component is safe and cheap to use in an experiment. The bioelectric potential varies hourly, so the analysis of the potential is carried out using a summation value for unit intervals, for example, a minute and half an hour. As a result, it is obvious that the absorption capability for the atmospheric component (ethyl alcohol) increases as the potential summation value increases. Major differences in absorption capability could not be recognized between the summation values for homogeneous and complex lights. However, the capability differs depending on the ethyl alcohol concentration. It is considered that the results of this research can be applied to a vegetation factory in the future, and that the production efficiency can be improved.

\section{Experimental Procedure}

Plants have many sensory functions; thus, it necessary that a steady condition is maintained during an experiment on plant bioelectric potential. Such an experiment is carried out in a 3001 acrylic chamber. The subject plant and various sensors are installed in the chamber. The bioelectric potential varies hourly rapidly. It is difficult to derive some features from this change. The potential is inputted every $0.1 \mathrm{~s}$ and its absolute value is used in the analysis. The summation values of the inputted absolute potentials per unit of time [for example, a minute $\left(v_{\mathrm{m} 1}\right)$, half an hour $\left(v_{\mathrm{m} 30}\right)$, and an hour $\left.\left(v_{\mathrm{h} 1}\right)\right]$ are adopted in this study. Two electrodes are connected to two neighboring leaves of the subject plant and the ground is the potted soil. The electrode material is aluminum $(0.3$ $\mathrm{cm}^{2}$ ) and the difference in voltage between the electrodes is amplified 100 times. The value is inputted into a low-pass filter that cuts off the frequency component over $10 \mathrm{~Hz}$. Then, it is inputted into an AD converter and data logger. Finally, it is inputted into a PC 
and analyzed. The following sensors are installed in the experimental chamber: tin oxide gas sensor (Figaro TGS\#800), temperature and humidity sensor with an atmospheric pressure sensor (T\&D, TR-73U), and illuminance sensor (T\&D, PHR-51). The gas sensor can be used to measure the concentration of ethyl alcohol. The following eight types of irradiation light are adopted in the experiment: darkness, red (660 nm), green $(525 \mathrm{~nm})$, and blue $(475 \mathrm{~nm})$, which are monochromatic lights, and white light, red and green, red and blue, and green and blue, which are mixed lights. LED panels (CCS Inc., ISL- $150 \times 150$ series) are used in the experiment as the irradiation light source. The experimental system used is shown in Fig. 1. In this figure, "Action" is performed depending on the "Growth model". That is, the environmental factors for the subject plant are monitored using a PC and the vegetation condition is identified. For example, fertilization as an action is carried out through an interface if necessary. At that time, the condition is coordinated with the optimum growth model and the best action is carried out. The action is derived by cross-checking with the vital phenomenon of the plant, which is a type of feedback. In the future, it is necessary to adopt "system biology", which can analyze plant vital phenomena as systems engineering.

White radish (scientific name: Raphanus sativus var. longipinnatus) is adopted as a subject plant. A photograph is shown in Fig. 2. It has many special characteristics (e.g., medicinal effect) and is consumed widely since early times. In particular, it is known to the Japanese and is served frequently. The moisture content of radish is about 95\%: therefore, adequate moisture in the potted soil should be considered in the experiment. Plants can absorb exhaust fumes in the atmosphere as a growth substance. In this investigation, the plant absorption capability $\left(P_{\mathrm{a}}\right)$ for ethyl alcohol is adopted as a parameter. The capability is defined as follows. First, ethyl alcohol is injected to reach a specified concentration using a microsyringe. The output of the gas sensor increases at the same time as the injection and reaches a peak level. After that, the output decreases

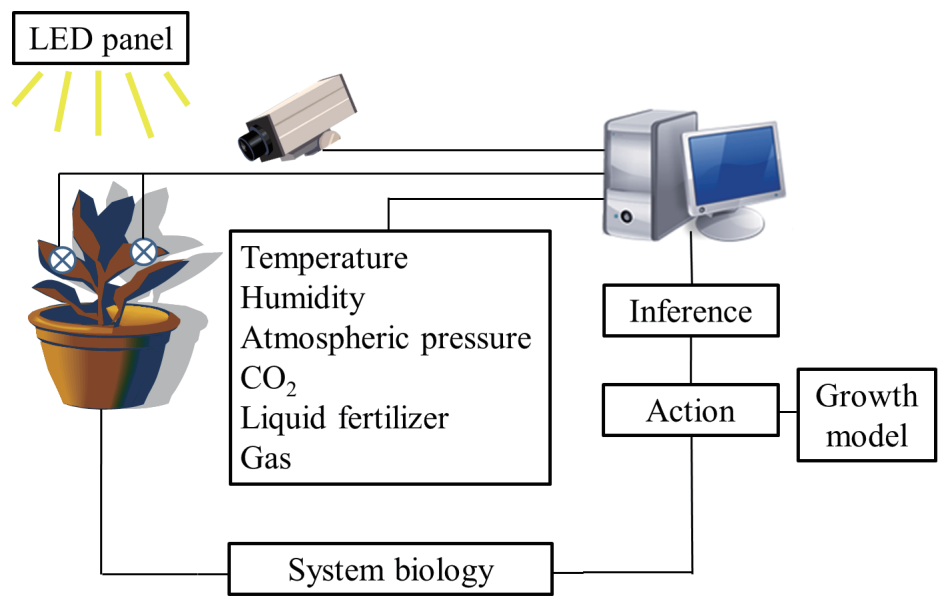

Fig. 1. (Color online) Experimental system. 


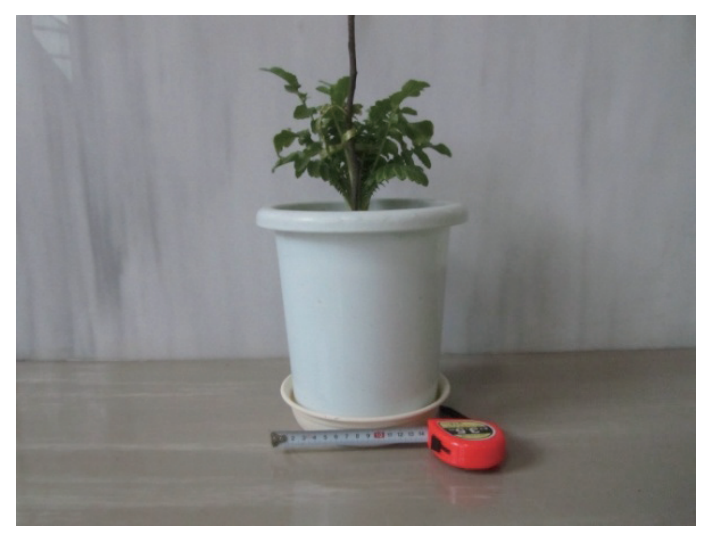

Fig. 2. (Color online) Photograph of subject plant (Japanese radish).

gradually owing to the absorption capability of the radish for the alcohol. The peak value $(h)$ from the offset level of the output is derived and the time from the injection to the half value of $h$ is measured, which is called the half width $\left(t_{\mathrm{w}}\right)$. The capability $\left(P_{\mathrm{a}}\right)$ is derived using $h$ and $t_{\mathrm{w}}$, and the equation can be represented as eq. (1). The relationship between $P_{\mathrm{a}}$ and $v_{\mathrm{m} 1}$ is introduced. The frequency of irradiation light is adopted as a parameter.

$$
P_{\mathrm{a}}=h / t_{\mathrm{w}} \times 100
$$

\section{Experimental Results}

\subsection{Output characteristic of gas sensor}

A tin oxide gas sensor is used to detect the concentration of ethyl alcohol in the experimental chamber. The sensor is sensitive to various types of reducing gas, that is, it does not have selectivity to a specific gas. The subject plant is installed in the chamber at 15:00 and ethyl alcohol is injected at 17:00 every experiment. The experiment is finished the next morning at 7:00. The data for 16:00 to 6:00 are used to analyze the plant characteristics. A typical gas sensor output characteristic is shown in Fig. 3. The concentration of ethyl alcohol is $15 \mathrm{ppm}$ and the irradiation light is red (intensity $73 \mathrm{~lx}$ ). The output increases rapidly until $0.313(\mathrm{~V})$. The offset level, which means the level right before the injection, is $0.0174 \mathrm{~V}$ and $h$ is $0.2956 \mathrm{~V}$. The absorption capability $\left(P_{\mathrm{a}}\right)$ is about $8.3(\mathrm{~V} / \mathrm{h})$ because $t_{\mathrm{w}}$ is $3.556 \mathrm{~h}$. The concentrations of $0,8,12$, and $15 \mathrm{ppm}$ are set in the experiments. The relationship between the sensor output $h$ and each concentration is shown in Fig. 4. It increases linearly as the concentration increases. The characteristic saturates over $25 \mathrm{ppm}$. The value of $P_{\mathrm{a}}$ can be derived until $20 \mathrm{ppm}$, which is the limit, and the plant is susceptible partially to high concentration. Thus, the experiment is carried out until $15 \mathrm{ppm}$. 

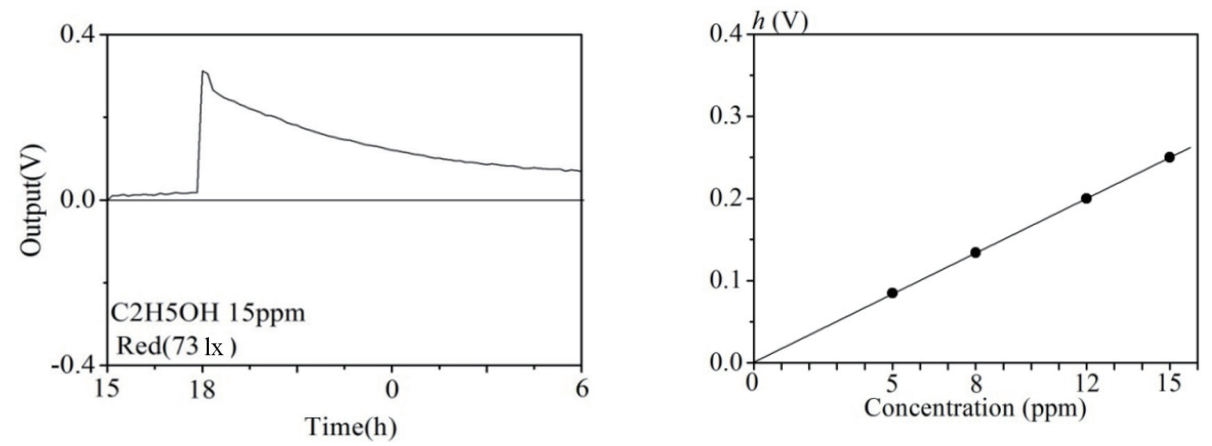

Fig. 3 (left). Typical gas sensor output characteristic.

Fig. 4 (right). Relationship between gas sensor output $h$ and ethyl alcohol concentration.

\subsection{Characteristic of bioelectric potential}

Two electrodes are connected to two neighboring leaves of the subject plant and the ground is the potted soil. The bioelectric potential, which is a differential voltage between the two electrodes, is inputted into a PC every $0.1 \mathrm{~s}$. This characteristic is indicated in Fig. 5. It is a unique characteristic of the subject plant illuminated with white light for $1 \mathrm{~min}$. It is difficult to derive some features in the figure because the characteristic oscillates sharply. Therefore, the summation value of the potential for 1 $\min \left(v_{\mathrm{m} 1}\right)$ is adopted for analyzing this plant characteristic and is shown in Fig. 6. The figure shows the $v_{\mathrm{m} 1}$ characteristic between 15:00 and 6:00 the next morning. In fact, the average value $\left(v_{\mathrm{m} 1 \text {-ave }}\right)$ of $v_{\mathrm{m} 1}$, which is located near the half width in the gas sensor characteristic, is adopted and analyzed. In addition, $v_{\mathrm{m} 30}$ and $v_{\mathrm{h} 1}$ are also adopted in the analysis. They indicate the summation values for $30 \mathrm{~min}$ and $1 \mathrm{~h}$. The $v_{\mathrm{m} 30}$ characteristic is shown in Fig. 7, and the $v_{\mathrm{h} 1}$ characteristic is also indicated in Fig. 8. These two characteristics correspond to that in Fig. 6, which is smoothed by expanding the summation time. In the figures, the characteristics are higher at the start and end of the experiment to some degree.

\subsection{Absorption characteristic of ethyl alcohol}

Plants grow by absorbing various gaseous substances in the atmosphere, that is, plants have a purifying capability. Moreover, they can take in nutrients through the roots. It is obvious that formaldehyde is taken in as a growth substance. ${ }^{(12-14)}$ Formaldehyde is a substance responsible for the sick house syndrome. It is thought that alcohol is also a nutrient. $P_{\mathrm{a}}$ is used as an absorbing capability index in this study. This index increases larger when the half width $\left(t_{\mathrm{w}}\right)$ of the gas sensor output decreases for the peak level, namely, the plant can absorb the chemical rapidly. The relationship between the bioelectric potential and the plant growth rate can be revealed by examining the relationship between $v_{\mathrm{ml}}$ and $P_{\mathrm{a}}$. The growth rate can be controlled using the relationship 

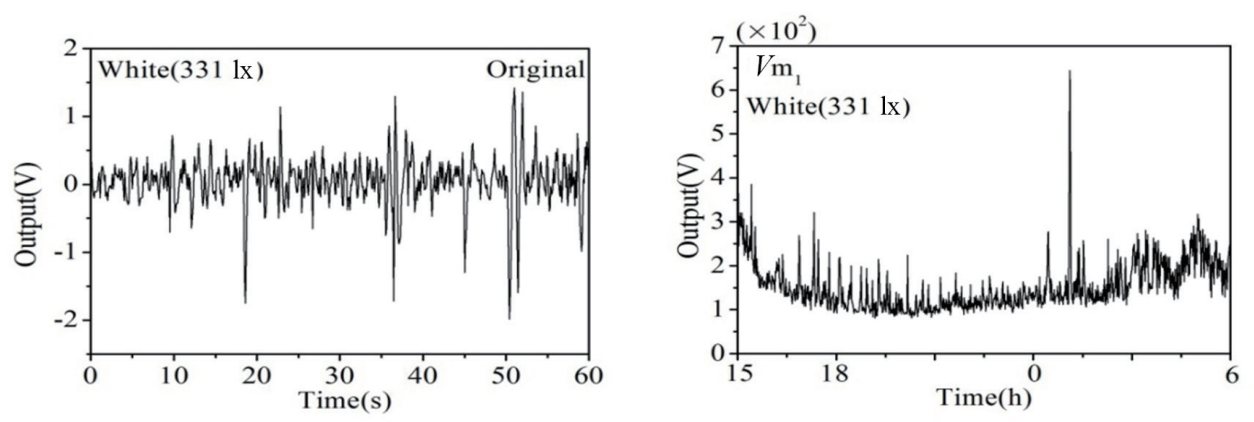

Fig. 5 (left). Original bioelectric potential of a Japanese radish for $1 \mathrm{~min}$.

Fig. 6 (right). $v_{\mathrm{m} 1}$ characteristic of Japanese radish from 15:00 to 6:00 the next morning.
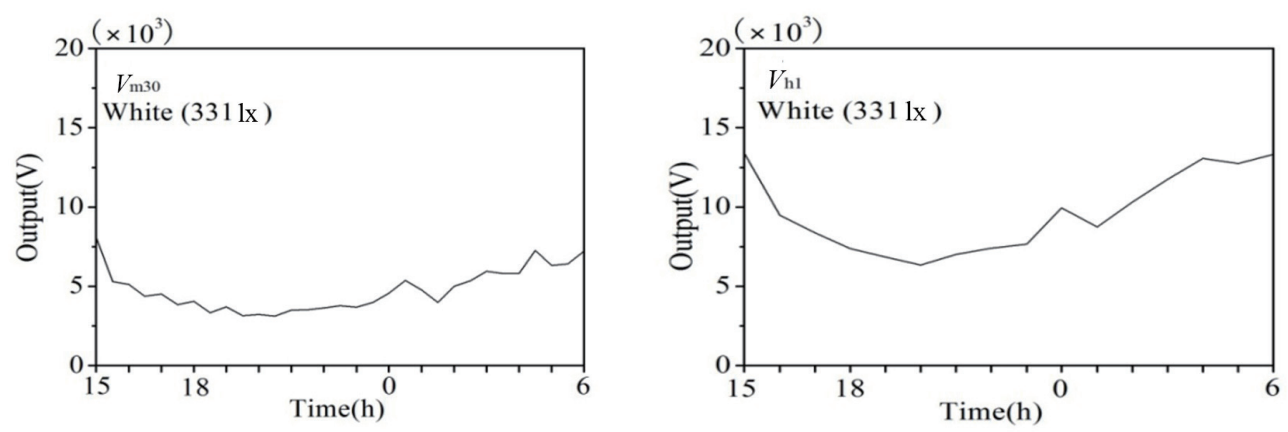

Fig. 7 (left). $v_{\mathrm{m} 30}$ characteristic of Japanese radish.

Fig. 8 (right). $v_{\mathrm{h} 1}$ characteristic of Japanese radish.

between $v_{\mathrm{m} 1}$ and $P_{\mathrm{a}}$ in a vegetable factory. The summed bioelectric potential near the half width $\left(t_{\mathrm{w}}\right)$ of the gas sensor characteristic is adopted to introduce some features. Every average bioelectric potential $\left(v_{\mathrm{m} 1}, v_{\mathrm{m} 30}\right.$, and $\left.v_{\mathrm{h} 1}\right)$ from the injection to the end of the experiment was compared with that near the half width under various experimental conditions. It became clear that the values are nearly equal with respect to each summation time; thus, the average values of $v_{\mathrm{m} 1}, v_{\mathrm{m} 30}$, and $v_{\mathrm{h} 1}$ near each half width can be used for analyzing the plant characteristics. There is a possibility that the taste and texture of vegetation change according to the gaseous species injected as nutrients. The relationship between $v_{\mathrm{ml}-\text { ave }}$ and $P_{\mathrm{a}}$ in $12 \mathrm{ppm}$ ethyl alcohol is revealed in Fig. 9. The correlation coefficient is $\gamma \approx 0.55$, and there is some degree of correlation. Similarly, the relationship between $v_{\mathrm{m} 30-\text { ave }}$ and $P_{\mathrm{a}}$ is shown in Fig. 10 and that between $v_{\mathrm{hl}-\text { ave }}$ and $P_{\mathrm{a}}$ is indicated in Fig. 11. Each horizontal axis means the average bioelectric potential near the half width of the gas sensor output, which represents the chemical concentration. It shows the relationship under eight types of irradiation light. The correlation coefficient 

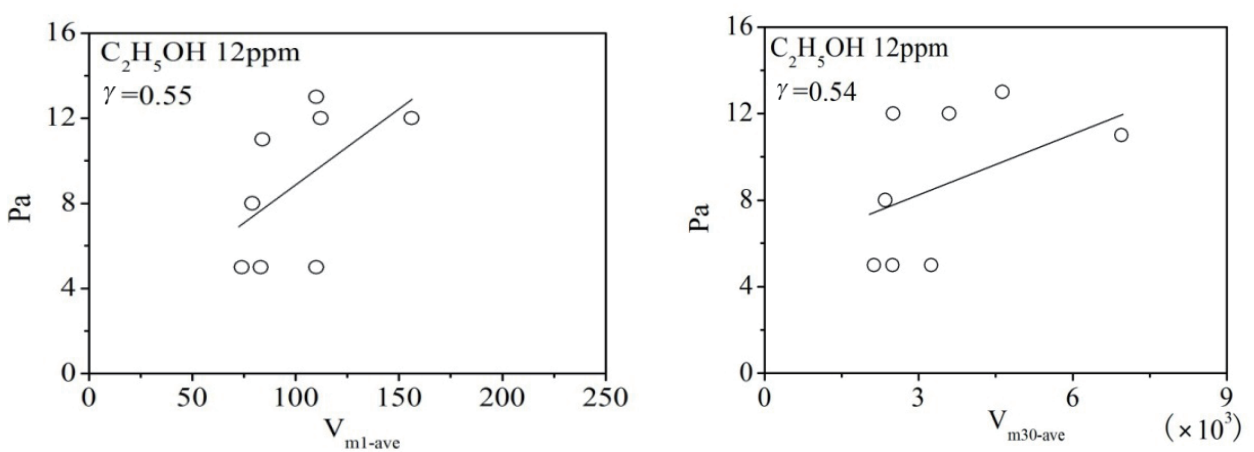

Fig. 9 (left). Relationship between $P_{\mathrm{a}}$ and $v_{\mathrm{ml} \text {-ave }}$ at $12 \mathrm{ppm}$ concentration.

Fig. 10 (right). Relationship between $P_{\mathrm{a}}$ and $v_{\mathrm{m} 30 \text {-ave }}$ at $12 \mathrm{ppm}$ concentration.

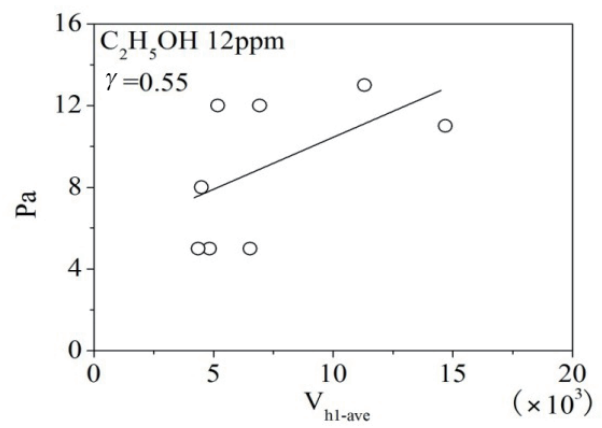

Fig. 11. Relationship between $P_{\mathrm{a}}$ and $v_{\mathrm{hl} \text {-ave }}$ at $12 \mathrm{ppm}$ concentration.

is $\gamma \approx 0.55$ in each case. The scattering plots in Figs. 10 and 11 closely resemble each other. Therefore, the potential and $P_{\mathrm{a}}$ could be indicated using the $v_{\mathrm{hl}-\text { ave }}$ characteristic. The average bioelectric potential near the half width could be used in analysis. The half width could also be estimated. This is an important advantage.

\subsection{Relationship between irradiation light and $\mathrm{P}_{a}$}

The vegetable physiology varies depending on the irradiation light. It is reported that the growth rate and taste of plants such as vegetables change owing to the quantity and frequency of the irradiation light. The bioelectric potential can be regarded as an effective sensor to detect the ripeness and freshness of fruits and vegetables. It became clear that the relationships between the potentials ( $v_{\mathrm{m} 1 \text {-ave }}, v_{\mathrm{m} 30 \text {-ave }}$, and $\left.v_{\mathrm{h} 1 \text {-ave }}\right)$ and the irradiation light frequency have positive correlations. In this section, $P_{\mathrm{a}}$ is examined under eight types of irradiation light. It is thought that $P_{\mathrm{a}}$ is related to the plant growth rate. It is clarified that the growth rate can be controlled by varying $P_{\mathrm{a}}$. However, a notable difference in potential is not desired. It is effective when a difference of several percent (5 to $6 \%$ ) in $P_{\text {a }}$ could be derived by the irradiation light frequency. 
The change in $P_{\mathrm{a}}$ is derived by varying the alcohol concentration in darkness and under three homogeneous lights. The result is shown in Fig. 12. In this figure, "R" indicates red light, " $G$ " indicates green light, and " $B$ " indicates blue light. "Black" is "in darkness". The experiment is carried out at three concentrations, namely, 8,12 , and $15 \mathrm{ppm}$. $P_{\mathrm{a}}$ tends to be minimum at $12 \mathrm{ppm}$ concentration under homogenous light. Its value in darkness is nearly the same as that under light. It could not be determined that $P_{\mathrm{a}}$ in ethyl alcohol atmosphere increases owing to the photosynthesis of white radish, which is a root crop. It is considered that plants have the capability to purify the atmosphere, and $P_{\mathrm{a}}$ increases with the concentration. Therefore, $P_{\mathrm{a}}$ becomes higher at a higher concentration. A similar experiment is carried out under mixed light. The result is shown in Fig. 13. It is maximum at $12 \mathrm{ppm}$ concentration. This result is different from that in Fig. 12. The variation is less under mixed light than under homogenous light. The coefficient of variation $(c v)$ of all the plots in Fig. 12 is 0.36 , and that in Fig. 13 is 0.16 . There is little change in $P_{\mathrm{a}}$ under white light, and $c v$ is small under mixed light. It is considered that plants can fulfill the absorption function for ethyl alcohol stably under mixed light. The function becomes stable by receiving mixed light, which includes various frequencies.

The histogram of $P_{\text {a }}$ shown in Figs. 12 and 13 is introduced, and the result is shown in Fig. 14. The vertical axis indicates the statistical frequency. The average $P_{\mathrm{a}}$ is 9.33 and the standard deviation $\sigma$ is $2.87 . c v$ is about 0.31 . It is relatively small and the plant fulfills the function stably. It is important to establish the light environment in which the plant can fulfill stable functions.
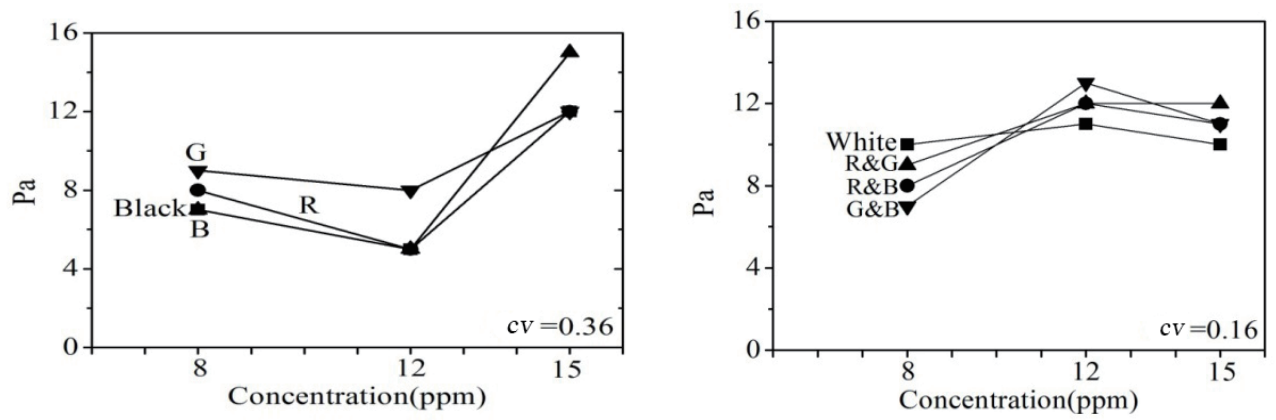

Fig. 12 (left). Absorption capacity $P_{\mathrm{a}}$ of Japanese radish for ethyl alcohol under monochromatic light.

Fig. 13 (right). Absorption capacity $P_{\mathrm{a}}$ of Japanese radish for ethyl alcohol under compound light. 


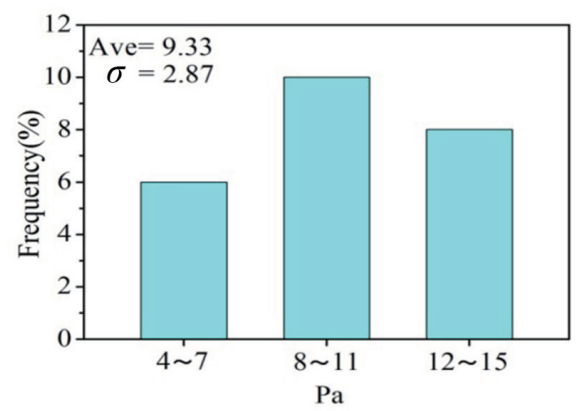

Fig. 14. (Color online) Histogram of $P_{\mathrm{a}}$ under all types of irradiation light.

\section{Conclusions}

White radish has been eaten from time immemorial and has a medicinal effect. It is a common vegetable in the world. In Japan, it is one of the seven herbs of spring and is widely consumed. It is also used as seasoning. It is categorized as a root crop and the white radish's fleshy root contains about $95 \%$ moisture. It is always necessary to provide fresh radish in the market. In this study, the relationship between the bioelectric potential and the absorption capability for ethyl alcohol is examined by producing radish in a plant factory. Suitable production management and cost consciousness in the factory are desired. Therefore, this study is conducted as a preliminary experiment to determine the plant characteristics that can be applied to the factory.

Our results show that the plant bioelectric potential varies hourly, and it was clarified that $v_{\mathrm{h} 1 \text {-ave }}$ can effectively indicate the characteristics, which means the summation value for one hour. $v_{\text {m1-ave }}$ and $v_{\text {m30-ave }}$, which mean the summation values for $1 \mathrm{~min}$ and half an hour, are also effective in deriving the bioelectric features. These values show a close correlation with absorption capability $\left(P_{\mathrm{a}}\right)$. The coefficient is about 0.55 , which is not very large. The absorption function is stable under mixed light compared with that under homogeneous light, and the value is about 10. It somewhat varies depending on the light frequency. There is a difference in potential because the atmospheric pressure and the vegetable physiology are different in each experiment. In each experiment, the conditions are fixed wherever possible. The temperature is about $26{ }^{\circ} \mathrm{C}$ and the relative humidity (RH) is over $95 \% \mathrm{RH}$ in the experimental chamber.

It is necessary to collect and accumulate bioelectric potential data from the standpoint of vegetable physiology. Many institutions have studied the microphysiology of plants. It is also necessary to investigate the macrophysiology when the production efficiency is increased. The production efficiency of vegetables will be increased by synergistic effects on plant physiology in the vegetable factory. 


\section{References}

1 D. Chamovitz: What a Plant Knows (Scientific American, New York, 2012) pp. 9-134.

2 T. Oyabu: J. Vac. Soc. Jpn. 55 (2012) 366 (in Japanese).

3 M. Tokuda, L. Shao, T. Oyabu and H. Nanto: IEEJ Trans. Sens. Micromachines 132 (2012) 143 (in Japanese).

4 K. Baosheng, S. Shibata, A. Sawada, T. Oyabu and H. Kimura: Sens. Mater. 21 (2009) 445.

5 F. Balska, S. Mancuso and D. Volkmann: Communication in Plants (Springer, New York, 2006) pp. 1-200.

6 C. Backster: Primary Perception (White Rose Millennium Press, California, 2003) pp. 21-42.

7 K. Ando, Y. Hasegawa, T. Yaji and H. Uchida: IEEJ Trans. Sens. Micromachines 132 (2012) 159 (in Japanese).

8 S. Arima: J. Soc. Insturum. Control. Engineers 50 (2011) 1037 (in Japanese).

9 R. A. Betts, O. Boucher, M. Collins, P. M. Cox, P. D. Falloon, N. Gedney, D. L. Hemming, C. Huntingford, C. D. Jones, D. M. H. Sexton and M. J. Webb: Nature 448/30 (2007) 1037.

10 B. H. Thewlis: New Phytol. 50 (1952) 263.

11 M. Takatuji: Plant Factory (Ohmsya, Tokyo 2010) pp. 1-21 (in Japanese).

12 P. Dingle, P. Tapsell and S. Hu: Environmental Contamination and Toxicology (Springer-Verlag, New York, 2000) pp. 302-308.

13 M. Giese, U. Bauer-Doranth, C. Langebartels and H. Sandermann Jr.: Plant Physiol. 104 (1994) 1301.

14 H. H. Tabak, S. A. Quave, C. I. Mashini, E. F. Barth: J. Water Pollut. Control Fed. 53 (1981) 1503. 